\title{
Determining Dark-Matter-Electron Scattering Rates from the Dielectric Function
}

\author{
Yonit Hochberg, ${ }^{1}$ Yonatan Kahn $\odot,{ }^{2,3}$ Noah Kurinsky $\odot,{ }^{4,5}$ Benjamin V. Lehmann $\odot,{ }^{6,7}$ \\ To Chin Yu $\odot,{ }^{8,9}$ and Karl K. Berggren ${ }^{10}$ \\ ${ }^{1}$ Racah Institute of Physics, Hebrew University of Jerusalem, Jerusalem 91904, Israel \\ ${ }^{2}$ Department of Physics, University of Illinois at Urbana-Champaign, Urbana, Illinois 61801, USA \\ ${ }^{3}$ Illinois Center for Advanced Studies of the Universe, University of Illinois at Urbana-Champaign, \\ Urbana, Illinois 61801, USA \\ ${ }^{4}$ Fermi National Accelerator Laboratory, Batavia, Illinois 60510, USA \\ ${ }^{5}$ Kavli Institute for Cosmological Physics, University of Chicago, \\ Chicago, Illinois 60637, USA \\ ${ }^{6}$ Department of Physics, University of California Santa Cruz, \\ Santa Cruz, California 95064, USA \\ ${ }^{7}$ Santa Cruz Institute for Particle Physics, Santa Cruz, California 95064, USA \\ ${ }^{8}$ Department of Physics, Stanford University, Stanford, California 94305, USA \\ ${ }^{9}$ SLAC National Accelerator Laboratory, 2575 Sand Hill Road, \\ Menlo Park, California 94025, USA \\ ${ }^{10}$ Department of Electrical Engineering and Computer Science, Massachusetts Institute of Technology, \\ Cambridge, Massachusetts 02139, USA
}

(Received 25 February 2021; accepted 2 September 2021; published 6 October 2021)

\begin{abstract}
We show that the rate for dark-matter-electron scattering in an arbitrary material is determined by an experimentally measurable quantity, the complex dielectric function, for any dark matter interaction that couples to electron density. This formulation automatically includes many-body effects, eliminates all systematic theoretical uncertainties on the electronic wave functions, and allows a direct calibration of the spectrum by electromagnetic probes such as infrared spectroscopy, x-ray scattering, and electron energyloss spectroscopy. Our formalism applies for several common benchmark models, including spinindependent interactions through scalar and vector mediators of arbitrary mass. We discuss the consequences for standard semiconductor and superconductor targets and find that the true reach of superconductor detectors for light mediators exceeds previous estimates by several orders of magnitude, with further enhancements possible due to the low-energy tail of the plasmon. Using a heavy-fermion superconductor as an example, we show how our formulation allows a rapid and systematic investigation of novel electron scattering targets.
\end{abstract}

DOI: 10.1103/PhysRevLett.127.151802

Dark-matter-electron scattering was first proposed for sub-GeV dark matter (DM) detection less than a decade ago [1], and there has been enormous theoretical [2-37] and experimental [38-51] progress since then. Since electrons are not free particles, but are bound in atoms or delocalized across solids, they have favorable kinematics for light DM scattering. However, the rich complexity of condensed matter systems complicates the calculation of scattering rates. Not only do bound electrons have different wave functions than their free-particle counterparts [52], many condensed matter systems exhibit collective electronic

Published by the American Physical Society under the terms of the Creative Commons Attribution 4.0 International license. Further distribution of this work must maintain attribution to the author(s) and the published article's title, journal citation, and DOI. Funded by SCOAP . modes such as plasmons [53]. A formalism describing DM scattering with a single electronic state [3,25] can potentially miss important electron interaction and correlation effects and must carefully account for "screening," where the electron density rearranges itself to partially cancel out DM-induced perturbations [6].

In this Letter, we propose to bypass the single-particle formulation entirely and frame the problem of DM-electron scattering in terms of matrix elements of the many-body electron density operator. This perspective is inspired by a classic paper on collective energy loss in solids [54], and since it does not rely on a particular choice of eigenstates, it is equally applicable to all systems: atoms, molecules, metals, insulators, or more exotic materials. Moreover, it intrinsically accounts for all electron interactions and correlations in the target by relating the scattering rate to an experimentally measurable quantity, the complex dielectric function $\epsilon(\mathbf{q}, \omega)$. Crucially, since $\epsilon(\mathbf{q}, \omega)$ is defined as a 
linear response function, the response of the target to a momentum transfer $\mathbf{q}$ and energy deposit $\omega$ is determined by density matrix elements that are the same whether measured by DM-electron scattering or by an electromagnetic probe [55,56]. The assumption of linear response applies as long as DM interactions are weaker than electromagnetism.

The key result of this Letter is that the total scattering rate for DM with mass $m_{\chi}$ and velocity $\mathbf{v}_{\chi}$ in an arbitrary target is given by

$$
\Gamma\left(\mathbf{v}_{\chi}\right)=\int \frac{d^{3} \mathbf{q}}{(2 \pi)^{3}}|V(\mathbf{q})|^{2}\left[2 \frac{q^{2}}{e^{2}} \operatorname{Im}\left(-\frac{1}{\epsilon\left(\mathbf{q}, \omega_{\mathbf{q}}\right)}\right)\right],
$$

where $\omega_{\mathbf{q}}=\mathbf{q} \cdot \mathbf{v}_{\chi}-\left(q^{2} / 2 m_{\chi}\right), q=|\mathbf{q}|, e$ is the electron charge, and $V(\mathbf{q})$ is the nonrelativistic DM-electron potential. The full derivation can be found in the Supplemental Material [57], and follows mainly from the arguments made in Ref. [54]. The target-dependent object that appears in the integrand,

$$
\mathcal{W}(\mathbf{q}, \omega) \equiv \operatorname{Im}\left(-\frac{1}{\epsilon(\mathbf{q}, \omega)}\right)=\frac{\operatorname{Im}[\epsilon(\mathbf{q}, \omega)]}{|\epsilon(\mathbf{q}, \omega)|^{2}}
$$

is known as the loss function. The only assumptions we have made about the DM interactions in deriving Eq. (1) are (i) that the nonrelativistic Hamiltonian coupling DM to electrons takes the form $\hat{H}_{\text {int }}=\sum_{i} V\left(\hat{\mathbf{r}}_{\chi}-\hat{\mathbf{r}}_{i}\right)$, depending only on the electron position operators $\hat{\mathbf{r}}_{i}$ and no other operators such as spin or momentum, and (ii) that $\hat{H}_{\text {int }}$ can be treated perturbatively.

The consequences of Eq. (1) are of immediate importance for DM-electron scattering. Spin-independent Hamiltonians arise in many common benchmark models, including those for scattering through scalar and vector mediators. The presence of $\mathcal{W}$ implies that all of these interactions are screened. The importance of screening was first noted for a kinetically mixed dark photon mediator in a solid-state target [6] and later for a scalar mediator [35]. Our results show that a scalar force that couples equally and oppositely to electrons and protons, whether short- or longranged, is screened exactly like a kinetically mixed dark photon. Furthermore, as long as ion contributions to the loss function are negligible (as in semiconductors well above the gap), forces that couple differently to nucleons and electrons are still screened identically. All such screening effects are invisible in a single-particle picture.

Since $\mathcal{W}(\mathbf{q}, \omega)$ is directly measurable through electromagnetic scattering, DM-electron scattering experiments can be calibrated experimentally, exactly as was done for DM absorption [65-67] using the measured real conductivity $\sigma_{1}(\omega)=(\omega / 4 \pi)|\epsilon(0, \omega)|^{2} \mathcal{W}(0, \omega)$. The advantage of our approach is that the loss function can also be modeled semianalytically in certain relevant energy and momentum regimes, and such models can be compared directly to data.
This enables rapid assessment of candidate experimental targets and potentially bypasses the need for numerical electron wave functions to determine the reach of novel detector materials. As shown in the Supplemental Material [57], the loss function contains a sum over all possible final states of the target; thus Eq. (1) represents the maximum possible scattering rate that could be observed at any experiment sensitive to a particular subset of excitations, for example, electron-hole pairs.

In the following sections, we show that, in a material with free carriers, the loss function scales as $\mathcal{W}(\mathbf{q}, \omega) \propto q$ at small $\omega$, which can be interpreted as the familiar screening that partially suppresses the $1 / q^{4}$ enhancement characteristic of a light mediator. We then show that, if $\mathcal{W}(0, \omega)$ is nonvanishing, a rate enhancement at small $q$ remains whenever $\omega$ is kinematically accessible. This behavior of the loss function can arise in two qualitatively different ways: interband transitions in insulators and longrange plasmons, which are generically present in all materials. As we will show, the low-energy plasmon tail may improve the sensitivity of superconducting detectors to light DM by several orders of magnitude, and materials with Fermi velocities slower than $v_{\chi}$ may allow DM to access the bulk of the loss function rather than the tail. We illustrate these kinematic regimes in Fig. 1.

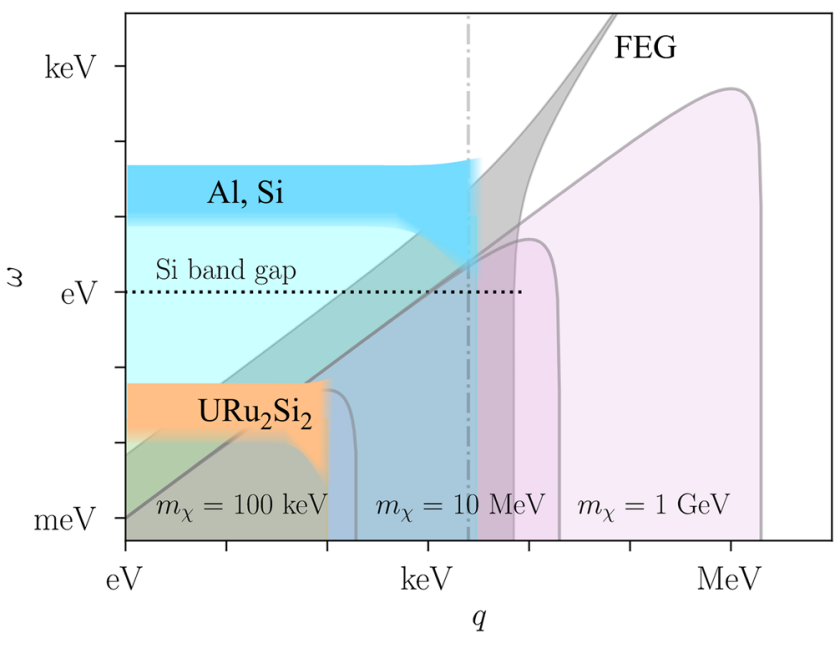

FIG. 1. Schematic depiction of the relevant kinematics for sub$\mathrm{GeV}$ DM. The shaded purple parabolas represent the kinematically allowed region of $q$ and $\omega$ for the labeled DM masses, as in Ref. [25], for a fixed DM speed $v_{\chi}=10^{-3}$, with upper boundary $\omega=q v_{\chi}$ independent of $m_{\chi}$. The blue and orange shaded regions represent the support of the plasmon part of the loss function. The tail extends into the DM region for conventional materials such as $\mathrm{Al}$ and $\mathrm{Si}$ and for heavy-fermion materials such as $\mathrm{URu}_{2} \mathrm{Si}_{2}$, the plasmon peak lies in the DM region. The range of support for the FEG loss function is shown in shaded gray and can be used to approximate the rate in both superconductors and semiconductors over a limited range of $\omega$. The dot-dashed vertical line indicates the size of the Brillouin zone $(q \approx 2.3 \mathrm{keV})$ of $\mathrm{Si}$, while the horizontal dashed line indicates the band gap above which electron scattering can produce ionization. 
In this Letter, we adopt a generic form for the potential $V(\mathbf{q})=V(q)=\left[g_{\chi} g_{e} /\left(q^{2}+m_{\phi, V}^{2}\right)\right]$, which is valid for DM coupling through a scalar mediator $\phi$ or vector $V$. We compute scattering rates by integrating Eq. (1) over the DM velocity distribution, for which we take the standard halo model (see Supplemental Material [57] for details). We frame our results in terms of a reference cross section $\bar{\sigma}_{e}=\left(\mu_{e \chi}^{2} / \pi\right)\left|V\left(q_{0}\right)\right|^{2}$, where $\mu_{e \chi}$ is the electron-DM reduced mass and $q_{0}=\alpha m_{e} \simeq 3.7 \mathrm{keV}$ is a reference momentum. We show results for a light mediator $m_{\phi, V}^{2} \ll q^{2}$, with heavy mediator results given in the Supplemental Material [57] (Fig. S6).

Conventional superconductors.-Reference [5] first proposed using superconducting metals such as aluminum (Al) as targets for DM-electron scattering. Reference [6] soon pointed out that long-range Coulomb forces among electrons would screen DM interactions if mediated by a kinetically mixed dark photon. This effect was incorporated by multiplying the free-particle matrix element by $1 /\left|\epsilon_{\mathrm{RPA}}(\mathbf{q}, \omega)\right|^{2}$, where $\epsilon_{\mathrm{RPA}}$ is the dielectric function of a free electron gas (FEG) in the random phase approximation (RPA) at zero temperature.

Even within RPA, our formalism identifies two important corrections to the DM interaction rate from Ref. [6]. First, all interactions coupling to electron density are screened, including a light scalar mediator and a vector mediator without kinetic mixing. This unifies the reach for all models considered in Ref. [6]. Second, the analytic structure of the loss function imposed by causality implies a particular choice of branch cut in $\epsilon_{\mathrm{RPA}}$ differing from that used in Ref. [6] (see Supplemental Material [57] for details).

The latter correction improves the projected sensitivity of conventional superconductor detectors to DM scattering through a light mediator by several orders of magnitude at low masses. We can understand this by examining $\epsilon_{\mathrm{RPA}}$ in the kinematic regime $q \ll k_{F}, \omega \ll q v_{F}$ relevant for sub$\mathrm{MeV}$ DM scattering near the Fermi surface, where $k_{F}$ is the Fermi momentum and $v_{F}$ is the Fermi velocity, respectively, $3.5 \mathrm{keV}$ and $6.8 \times 10^{-3}$ in Al. The result is [68]

$$
\epsilon_{\mathrm{RPA}}(\mathbf{q}, \omega) \approx \frac{\lambda_{\mathrm{TF}}^{2}}{2 q^{2}}+i \frac{3 \pi \omega_{p}^{2} \omega}{2 q^{3} v_{F}^{3}},
$$

where $\lambda_{\mathrm{TF}} \simeq 3.8 \mathrm{keV}$ is the Thomas-Fermi screening length and $\omega_{p} \simeq 15 \mathrm{eV}$ is the plasma frequency. The imaginary part is typically smaller than the real part, so $\mathcal{W}(\mathbf{q}, \omega)$ scales as $\left[\left(\omega / q^{3}\right) /\left(1 / q^{4}\right)\right] \sim \omega q$, a much softer screening than the $q^{4}$ implied from $1 /|\epsilon|^{2}$.

Moving beyond RPA, we use the results of Ref. [69], which fits to data a model containing both a one-loop "local field" correction to the electron vertex and a $q$-dependent plasmon width $\Gamma_{p} / \omega_{p} \simeq 0.1-0.3$. The fit implies that the contribution from the ion polarizability in $\mathrm{Al}$ is small,

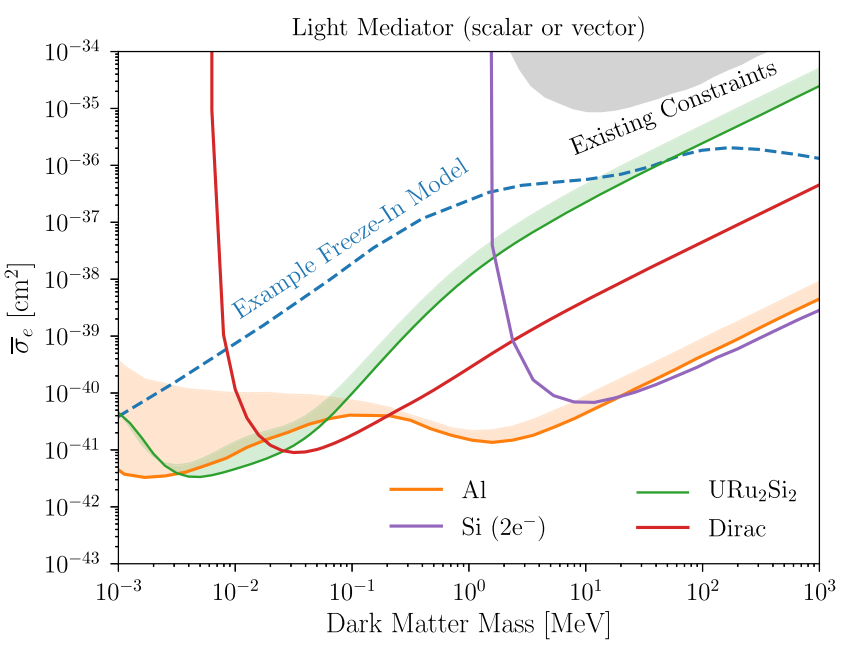

FIG. 2. The projected three-event reach of a 1-kg-yr exposure target of $\mathrm{Al}$ (orange), $\mathrm{Si}$ (purple), and $\mathrm{URu}_{2} \mathrm{Si}_{2}$ (green), computed for a light scalar or vector mediator using Eq. (1). For Al, the solid line uses $\mathcal{W}$ from Ref. [69], and the top of the shaded region uses the FEG model, both with $\omega \in[1 \mathrm{meV}, 1 \mathrm{eV}]$. The $\mathrm{URu}_{2} \mathrm{Si}_{2}$ loss function is taken from Ref. [70] with $\omega \in[1,74] \mathrm{meV}$, and the shaded region spans $\mathcal{W}$ measured along two crystal axes. $\mathrm{Si}$ is treated as a FEG with a $2 e^{-}$threshold, using the ionization model of Ref. [3]. We also show the reach for a Dirac material with density $10 \mathrm{~g} / \mathrm{cm}^{3}$, gap $2 \Delta=20 \mathrm{meV}$, Fermi velocity $v_{F}=4 \times 10^{-4}$, background dielectric constant $\kappa=40$, and Dirac band cutoff $\omega_{\max }=0.5 \mathrm{eV}$ (red); existing constraints from SENSEI [49], SuperCDMS HVeV [51], DAMIC [47], Xenon10 [14], DarkSide-50 [43], and Xenon1T [48] (shaded gray); and the theory target of a freeze-in model when the mediator is a kinetically mixed dark photon [1,71-73] (dashed blue). The corresponding plot for a heavy mediator is shown in the Supplemental Material [57] (Fig. S6).

justifying our approximation that only electrons contribute to the loss function. The projected reach for a $1 \mathrm{meV}$ threshold is shown in Fig. 2 for a light mediator, with comparisons to previous results given in Fig. S5 of the Supplemental Material [57]. The orange band reflects theoretical uncertainty in the proper form of the loss function in the energy range of interest (see Supplemental Material [57]).

In most materials, the loss function features a plasmon with a Lorentzian line shape peaked at $\omega_{p}[53,55]$ and a low-energy tail (see Fig. 1 and the Supplemental Material [57]). In the parametrization of Ref. [69], $\mathcal{W}(q=0, \omega)$ scales linearly with $\omega$ for $\omega \ll \omega_{p}$, and the plasmon tail dominates over the RPA contribution. Our results suggest that a kilogram-year exposure of an $\mathrm{Al}$ target with a $1 \mathrm{meV}$ threshold is sufficient to cover the entire freeze-in thermal relic target [1,71-73] above $10 \mathrm{keV}$. However, this depends on the extrapolation of the plasmon tail to $\mathrm{meV}$ energies, and existing measurements only characterize the loss function at $\omega \gtrsim 100 \mathrm{meV}$ [74]. Thus, additional measurements of $\mathcal{W}$ are crucial to accurately determine the sensitivity. There may also be contributions to $\mathcal{W}$ from 
coherent scattering with the Cooper pair condensate for energies $\omega \simeq 2 \Delta$, as well as finite-temperature effects. We leave investigation of these effects for future work [75].

Semiconductors. - In a typical semiconductor like silicon (Si) with a gap $E_{g} \sim \mathrm{eV}$, an energy deposit $\omega \simeq E_{g}$ requires a momentum deposit $q \geq E_{g} / v_{\chi} \sim \mathrm{keV}$ for $v_{\chi} \sim 10^{-3}$, independent of the DM mass, as shown in Fig. 1. The size of the first Brillouin zone in $\mathrm{Si}$ is $2 \pi / a \simeq 2 \mathrm{keV}$, where $a$ is the lattice constant. Thus, for $\omega \gtrsim 2 \mathrm{eV}$, DM is probing interatomic distances rather than delocalized electrons, and the electrons may be modeled as a FEG with an effective $k_{F} \simeq 2 \pi / a$ set by the total valence electron density. This approximation is an excellent match to both density functional theory (DFT) calculations [76] and data [77] for $q \simeq$ $5 \mathrm{keV}$ and $\omega \gg E_{g}$ in Si [78]; for sufficiently large $q$ ( $\sim 15 \mathrm{keV}$, see Supplemental Material [57]), the bound electron orbitals give large-momentum tails not captured by the FEG.

Equation (1) and Fig. 1 show that, at fixed $\omega$, the rate receives contributions from $\mathcal{W}(\mathbf{q}, \omega)$ over many orders of magnitude in $q$ for $m_{\chi} \gtrsim 10 \mathrm{MeV}$, so the FEG approximation is best for a light mediator, where $V(q) \propto q^{-4}$ weights the integrand most toward small $q$. Our formalism thus suggests a generic explanation for the behavior of the DM-electron spectrum in the 5-15 eV range (2-4 electronhole pairs in $\mathrm{Si}$ [3]) from light mediator exchange in any conventional semiconductor. The projected reach in $\mathrm{Si}$ under the FEG approximation with a $2 e^{-}$threshold is shown in Fig. 2 for a light mediator.

The differences among various targets become most apparent when $\omega \simeq E_{g}$, where the band structure describing delocalized electrons with $q \lesssim 2 \pi / a$ becomes important. In addition to band structure effects, there is also an irreducible contribution from the plasmon [79], where the tail extends into the kinematically allowed region for DM. This has important implications for rate predictions in currently operating semiconductor detectors [49-51]. DFT calculations predict a rate that peaks in the one- or two-electron bin, corresponding to $\omega \lesssim 8.3 \mathrm{eV}$, for all DM masses for which these energies are kinematically accessible [3]. Currently available measurements of $\mathcal{W}$ suggest the true rate in these few-electron bins may be somewhat larger. Near-gap effects are quite difficult to model [78], but in our formalism, they can be accounted for by making more precise measurements at $\omega \simeq E_{g}$ and $q \simeq E_{g} / v_{\chi}$.

On the other hand, for near-gap scattering in a narrowgap semiconductor $\left(E_{g} \sim 10 \mathrm{meV}\right)$, we have $q_{\min } \simeq$ $10 \mathrm{eV} \ll 2 \pi / a$, so the delocalized electrons in the uppermost valence band dominate the behavior of the scattering rate as $q \rightarrow 0$. We may understand the absence of screening in these systems through the Lindhard form of the dielectric function [68], which shows that $\epsilon(\mathbf{q}, \omega)$ has a finite limit as $\mathbf{q} \rightarrow 0$, with the imaginary part proportional to the interband transition matrix element. The lack of mobile charge carriers inhibits the screening present in metals. In the next section, we discuss an example of such a narrow-gap semiconductor: a Dirac material.

Novel materials.-Our formalism suggests that optimal materials for sub-GeV DM detection will have a loss function with large support for $\omega<v_{\chi} q$ (Fig. 1). For an ordinary metal with an electron effective mass $m^{*}=m_{e}$, the loss function is maximized at large $q$ when $\omega=q v_{F}$, where $v_{F}=k_{F} / m^{*} \simeq 10 v_{\chi}$. This is outside of the kinematically allowed region for DM scattering. For small $q$, collective modes such as the plasmon will dominate, but the plasmon is damped at momenta $q>q_{c} \simeq \omega_{p} / v_{F}$ [68] due to decay into the particle-hole continuum. Therefore, DM can only excite the undamped plasmon if $v_{\chi}>v_{F}$ [32]. Here we explore two qualitatively different ways to achieve $v_{F}<v_{\chi}$ : Dirac materials, in which $v_{F}$ is not tied directly to free-electron properties, and heavy-fermion materials, where strongly correlated electrons can create a Fermi surface with a large $m^{*}$.

Dirac materials, characterized by linear electronic dispersion $\omega(k)=v_{F} k$ with widely varying $v_{F}$ across materials [80], are promising targets for DM detection $[16,20,27,28]$. Consider a gapless isotropic Dirac material with a single Dirac cone and effective background dielectric constant $\kappa \equiv \operatorname{Re}[\epsilon(0,0)]$. In typical materials, $\operatorname{Re}(\epsilon) \gg$ $\operatorname{Im}(\epsilon)$ over the relevant $\mathbf{q}$ and $\omega$ [27], and we may write the loss function as

$\mathcal{W}_{\text {Dirac }}(q, \omega)=\frac{e^{2}}{12 \pi \kappa^{2} v_{F}} \Theta\left(\omega-v_{F} q\right) \Theta\left(\omega_{\max }-\omega\right)$.

The loss function with a gap $2 \Delta$ is given in the Supplemental Material [57]; $\mathcal{W}_{\text {Dirac }}(q, \omega)$ is constant as $q \rightarrow 0$ for all $\omega>2 \Delta$, as anticipated. The loss function immediately displays two key features of scattering in Dirac materials [16]: small $v_{F}$ increases the rate, and scattering is forbidden if $v_{\chi}<v_{F}$ for $\omega=\omega_{\mathbf{q}}$. In Fig. 2, we show the sensitivity of an isotropic Dirac material for a light mediator.

This analysis neglects many-body effects, including the plasmon contribution to the loss function. Dirac materials are expected to exhibit two tunable plasmon modes distinct from the ordinary valence plasmon: a temperaturedependent mode, which could lie in the $\mathcal{O}(\mathrm{meV})$ range [81-83], and a zero-temperature mode tunable with chemical potential [84]. Therefore, measurements of the loss function in real materials are crucial to accurately estimate the scattering rate, since the plasmon contribution may dominate [85] as was the case for superconductors.

Another way to lower $v_{F}$ is to find materials with ordinary quadratic dispersion but large effective masses. As an example, a number of materials containing $f$ electrons are known as heavy-fermion systems because they display a Fermi surface with $m^{*} \sim(10-100) m_{e}$ [86-88]. These materials are expected to have a plasmon at energy $\omega_{p}^{*} \simeq T^{*}$, the Fermi temperature of the heavy 
electrons [89]. One such material is $\mathrm{URu}_{2} \mathrm{Si}_{2}$, a heavyfermion superconductor with $T^{*}=75 \mathrm{~K}=6.5 \mathrm{meV}$ and $m^{*} \simeq 6 m_{e}$ [90], from which one may estimate $v_{F} \simeq$ $6.5 \times 10^{-5}, \quad \omega_{p}^{*} \simeq T^{*}=6.5 \mathrm{meV}, \quad$ and $\quad q_{c} \simeq \omega_{p}^{*} / v_{F} \simeq$ $100 \mathrm{eV}$. In reality, the measured loss function in $\mathrm{URu}_{2} \mathrm{Si}_{2}$ [70] shows considerable anisotropy with Lorentzian peaks at either 4or $6 \mathrm{meV}$ depending on the direction of $\mathbf{q}$, as well as a broad peak around $18 \mathrm{meV}$, which can also be interpreted as a heavy-fermion plasmon (see Supplemental Material [57]). Despite the extremely rich electron dynamics in this material, in our formalism we may compute the DM rate unambiguously once $\mathcal{W}$ is measured in the relevant kinematic regime.

The measured data (see Supplemental Material [57], Fig. S1) show that $\mathcal{W}(\omega) \propto \omega$ above the heavy-fermion plasmon peaks, consistent with the tail of the ordinary valence electron plasmon. However, in contrast to spectra from conventional superconductors or semiconductors, the measured loss function in $\mathrm{URu}_{2} \mathrm{Si}_{2}$ shows rich structure, which could be used to separate signals from backgrounds not due to fast-particle scattering. Integrating over $\omega$ from a threshold of $1 \mathrm{meV}$ up to $\omega_{\max }=74 \mathrm{meV}$, the maximum value where data exist, we obtain the projected reach in Fig. 2. The band spans measurements of $\mathcal{W}(\mathbf{q}, \omega)$ as $\mathbf{q} \rightarrow 0$ along two different crystal axes. We leave a full analysis of the anisotropic response to future work [75]. As expected, the reach in $\mathrm{URu}_{2} \mathrm{Si}_{2}$ can surpass $\mathrm{Al}$ in the mass range 5-40 keV, where the DM kinetic energy is comparable to the heavy-fermion plasmon energies. Our reach estimates motivate further study of $\mathrm{URu}_{2} \mathrm{Si}_{2}$ and similar materials as targets for light DM scattering.

Implications for experiments. - The advantage of our formulation of the DM scattering rate is that no theoretical input from, e.g., DFT is required to compute the scattering rate; the DM energy-loss spectrum from spin-independent electron scattering may be precisely predicted from a measurement with an electromagnetic probe in the appropriate kinematic regime. For MeV-GeV DM, x-ray scattering covers the regime $q \sim \mathrm{keV}$ and $\omega \sim \mathrm{eV}$ [56], while for $\mathrm{keV}-\mathrm{MeV} \mathrm{DM}$, momentum-resolved electron energy-loss spectroscopy can cover $q \sim \mathrm{eV}$ and $\omega \sim \mathrm{meV}$ [55,91]. These techniques are standard in condensed matter physics, and a rich literature on measurements of dielectric and loss functions already exists for a number of systems of interest.

The downside of this formalism is that it does not directly predict how many electron-hole pairs are created in the material per unit deposited energy, or how the energy is down-converted from plasmon excitations to charge and phonons. However, if individual quasiparticle contributions to $\mathcal{W}(\mathbf{q}, \omega)$ can be modeled, this information can be reconstructed. (For related work in the context of superconducting targets, see Ref. [92].) Moreover, the quasiparticle contributions may be determined empirically by correlating scattering events using an electromagnetic probe with the partition of excitations read out by the detector, as has been done for nuclear recoil calibrations at higher energy. We argue that these measurements should be considered the primary calibration mechanisms for DMelectron scattering, analogous to photoabsorption for bosonic DM absorption [65-67].

Finally, our work may be applied to unify the electronic and phonon descriptions of DM scattering with other subgap loss mechanisms that have not yet been explored, such as dielectric heating in insulators or coherent scattering off the superconducting condensate. Dielectric skin depth in the long-wavelength limit $\mathbf{q} \rightarrow 0$ is proportional to $\sqrt{\operatorname{Re}[\epsilon(\omega)]} /\{\omega \operatorname{Im}[\epsilon(\omega)]\}$, and thus materials with a small skin depth for terahertz photons and calorimetric readout should respond efficiently to DM-electron scattering, even for meV-scale energy deposits below the eV-scale electronic band gaps. Many materials have terahertz absorption features, so high-resolution terahertz or infrared transmission spectra are likely fertile ground for exploring new materials for $\mathrm{keV}$-scale DM scattering.

We thank Carlos Blanco, Vinayak Dravid, Rouven Essig, Sinéad Griffin, Adolfo Grushin, David Huse, Simon Knapen, Belina von Krosigk, Jonathan Kozaczuk, Eric David Kramer, Tongyan Lin, Mariangela Lisanti, Andrea Mitridate, Lucas Wagner, and Kathryn Zurek for enlightening discussions. Y. K. is indebted to Peter Abbamonte for relentlessly (and correctly) emphasizing the importance of the loss function and plasmon excitations for dark matter scattering. The idea for this work was conceived via an email exchange during the New Directions in Light Dark Matter workshop at Fermilab, supported by the Gordon and Betty Moore Foundation and the American Physical Society, and via a Skype call taken during the workshop "Quantum Information and Systems for Fundamental Physics" at the Aspen Center for Physics, which is supported by National Science Foundation Grant No. PHY-1607611. This project was supported in part by the Fermi National Accelerator Laboratory, managed and operated by Fermi Research Alliance, LLC under Contract No. DE-AC02-07CH11359 with the U.S. Department of Energy, through the Office of High Energy Physics QuantISED program. The work of Y. H. is supported by the Israel Science Foundation (Grant No. 1112/17), by the Binational Science Foundation (Grant No. 2016155), by the I-CORE Program of the Planning Budgeting Committee (Grant No. 1937/12), and by the Azrieli Foundation. The work of Y. K. is supported in part by DOE Award No. DE-SC0015655. Parts of this document were prepared by N. K. using the resources of the Fermi National Accelerator Laboratory (Fermilab), a U.S. Department of Energy, Office of Science, HEP User Facility. Fermilab is managed by Fermi Research Alliance, LLC (FRA), acting under Contract No. DEAC02-07CH11359. The work of B. V. L. is supported in part by DOE Award No. DE-SC0010107. T.C. Y. is supported by the U.S. Department of Energy under Award No. DE-AC02-76SF00515. K. K. B. acknowledges 
support for the later stages of the work from the Fermi Research Alliance, LLC (FRA) and the U.S. Department of Energy (DOE) under contract No. DE-AC02-07CH11359; the initial stages of the work were supported by the DOE under the QuantiSED program, Award No. DESC0019129.

Note added.-Similar work recently appeared in Ref. [93], which also discusses the loss function as a tool for predicting DM scattering rates. Our main results with respect to the loss function are substantively similar, although Ref. [93] emphasizes comparisons with ab initio methods, whereas the present Letter emphasizes the utility of the loss function formalism in target selection for future experiments.

[1] R. Essig, J. Mardon, and T. Volansky, Direct detection of sub-GeV dark matter, Phys. Rev. D 85, 076007 (2012).

[2] P. W. Graham, D. E. Kaplan, S. Rajendran, and M. T. Walters, Semiconductor probes of light dark matter, Phys. Dark Universe 1, 32 (2012).

[3] R. Essig, M. Fernandez-Serra, J. Mardon, A. Soto, T. Volansky, and T.-T. Yu, Direct detection of sub-GeV dark matter with semiconductor targets, J. High Energy Phys. 05 (2016) 046.

[4] S. K. Lee, M. Lisanti, S. Mishra-Sharma, and B. R. Safdi, Modulation effects in dark matter-electron scattering experiments, Phys. Rev. D 92, 083517 (2015).

[5] Y. Hochberg, Y. Zhao, and K. M. Zurek, Superconducting Detectors for Superlight Dark Matter, Phys. Rev. Lett. 116, 011301 (2016).

[6] Y. Hochberg, M. Pyle, Y. Zhao, and K. M. Zurek, Detecting superlight dark matter with fermi-degenerate materials, J. High Energy Phys. 08 (2016) 057.

[7] J. Alexander et al., in Dark Sectors 2016 Workshop: Community Report (2016) [arXiv:1608.08632].

[8] S. Derenzo, R. Essig, A. Massari, A. Soto, and T.-T. Yu, Direct detection of sub-GeV dark matter with scintillating targets, Phys. Rev. D 96, 016026 (2017).

[9] Y. Hochberg, Y. Kahn, M. Lisanti, C. G. Tully, and K. M. Zurek, Directional detection of dark matter with twodimensional targets, Phys. Lett. B 772, 239 (2017).

[10] B. J. Kavanagh, R. Catena, and C. Kouvaris, Signatures of Earth-scattering in the direct detection of dark matter, J. Cosmol. Astropart. Phys. 01 (2017) 012.

[11] T. Emken, C. Kouvaris, and I. M. Shoemaker, Terrestrial effects on dark matter-electron scattering experiments, Phys. Rev. D 96, 015018 (2017).

[12] T. Emken and C. Kouvaris, DaMaSCUS: The impact of underground scatterings on direct detection of light dark matter, J. Cosmol. Astropart. Phys. 10 (2017) 031.

[13] M. Battaglieri et al., US Cosmic Visions: New Ideas in Dark Matter 2017: Community Report, in U.S. Cosmic Visions: New Ideas in Dark Matter (2017) [arXiv:1707.04591].

[14] R. Essig, T. Volansky, and T.-T. Yu, New constraints and prospects for sub-GeV dark matter scattering off electrons in xenon, Phys. Rev. D 96, 043017 (2017).
[15] G. Cavoto, F. Luchetta, and A. Polosa, Sub-GeV dark matter detection with electron recoils in carbon nanotubes, Phys. Lett. B 776, 338 (2018).

[16] Y. Hochberg, Y. Kahn, M. Lisanti, K. M. Zurek, A. G. Grushin, R. Ilan, S. M. Griffin, Z.-F. Liu, S. F. Weber, and J. B. Neaton, Detection of sub-MeV dark matter with threedimensional Dirac materials, Phys. Rev. D 97, 015004 (2018).

[17] R. Essig, M. Sholapurkar, and T.-T. Yu, Solar neutrinos as a signal and background in direct-detection experiments searching for sub-GeV dark matter with electron recoils, Phys. Rev. D 97, 095029 (2018).

[18] T. Emken and C. Kouvaris, How blind are underground and surface detectors to strongly interacting dark matter?, Phys. Rev. D 97, 115047 (2018).

[19] Y. Ema, F. Sala, and R. Sato, Light Dark Matter at Neutrino Experiments, Phys. Rev. Lett. 122, 181802 (2019).

[20] R. M. Geilhufe, B. Olsthoorn, A. Ferella, T. Koski, F. Kahlhoefer, J. Conrad, and A. V. Balatsky, Materials informatics for dark matter detection, Phys. Status Solidi RRL 12, 1800293 (2018).

[21] D. Baxter, Y. Kahn, and G. Krnjaic, Electron ionization via dark matter-electron scattering and the Migdal effect, Phys. Rev. D 101, 076014 (2020).

[22] R. Essig, J. Pradler, M. Sholapurkar, and T.-T. Yu, Relation between the Migdal Effect and Dark Matter-Electron Scattering in Isolated Atoms and Semiconductors, Phys. Rev. Lett. 124, 021801 (2020).

[23] T. Emken, R. Essig, C. Kouvaris, and M. Sholapurkar, Direct detection of strongly interacting sub-GeV dark matter via electron recoils, J. Cosmol. Astropart. Phys. 09 (2019) 070.

[24] Y. Hochberg, I. Charaev, S.-W. Nam, V. Verma, M. Colangelo, and K. K. Berggren, Detecting Sub-GeV Dark Matter with Superconducting Nanowires, Phys. Rev. Lett. 123, 151802 (2019).

[25] T. Trickle, Z. Zhang, K. M. Zurek, K. Inzani, and S. Griffin, Multi-channel direct detection of light dark matter: Theoretical framework, J. High Energy Phys. 03 (2020) 036.

[26] S. M. Griffin, K. Inzani, T. Trickle, Z. Zhang, and K. M. Zurek, Multichannel direct detection of light dark matter: Target comparison, Phys. Rev. D 101, 055004 (2020).

[27] A. Coskuner, A. Mitridate, A. Olivares, and K. M. Zurek, Directional dark matter detection in anisotropic dirac materials, Phys. Rev. D 103, 016006 (2021).

[28] R. M. Geilhufe, F. Kahlhoefer, and M. W. Winkler, Dirac materials for sub-MeV dark matter detection: New targets and improved formalism, Phys. Rev. D 101, 055005 (2020).

[29] R. Catena, T. Emken, N. A. Spaldin, and W. Tarantino, Atomic responses to general dark matter-electron interactions, Phys. Rev. Research 2, 033195 (2020).

[30] C. Blanco, J. I. Collar, Y. Kahn, and B. Lillard, Dark matterelectron scattering from aromatic organic targets, Phys. Rev. D 101, 056001 (2020).

[31] N. A. Kurinsky, T. C. Yu, Y. Hochberg, and B. Cabrera, Diamond detectors for direct detection of sub-GeV dark matter, Phys. Rev. D 99, 123005 (2019).

[32] N. Kurinsky, D. Baxter, Y. Kahn, and G. Krnjaic, Dark matter interpretation of excesses in multiple direct detection experiments, Phys. Rev. D 102, 015017 (2020). 
[33] S. M. Griffin, Y. Hochberg, K. Inzani, N. Kurinsky, T. Lin, and T. C. Yu, Silicon carbide detectors for sub-GeV dark matter, Phys. Rev. D 103, 075002 (2021).

[34] A. Radick, A.-M. Taki, and T.-T. Yu, Dependence of dark matter-electron scattering on the galactic dark matter velocity distribution, J. Cosmol. Astropart. Phys. 02 (2021) 004.

[35] G. B. Gelmini, V. Takhistov, and E. Vitagliano, Scalar direct detection: In-medium effects, Phys. Lett. B 809, 135779 (2020).

[36] T. Trickle, Z. Zhang, and K. M. Zurek, Effective field theory of dark matter direct detection with collective excitations, arXiv:2009.13534.

[37] P. Du, D. Egana-Ugrinovic, R. Essig, and M. Sholapurkar, Sources of low-energy events in low-threshold dark matter detectors, arXiv:2011.13939.

[38] R. Essig, A. Manalaysay, J. Mardon, P. Sorensen, and T. Volansky, First Direct Detection Limits on Sub-GeV Dark Matter from XENON10, Phys. Rev. Lett. 109, 021301 (2012).

[39] J. Tiffenberg, M. Sofo-Haro, A. Drlica-Wagner, R. Essig, Y. Guardincerri, S. Holland, T. Volansky, and T.-T. Yu (SENSEI Collaboration), Single-Electron and SinglePhoton Sensitivity with a Silicon Skipper CCD, Phys. Rev. Lett. 119, 131802 (2017).

[40] R. Romani et al., Thermal detection of single $e-h$ pairs in a biased silicon crystal detector, Appl. Phys. Lett. 112, 043501 (2018).

[41] M. Crisler, R. Essig, J. Estrada, G. Fernandez, J. Tiffenberg, M. S. Haro, T. Volansky, and T.-T. Yu (SENSEI Collaboration), SENSEI: First Direct-Detection Constraints on subGeV Dark Matter from a Surface Run, Phys. Rev. Lett. 121, 061803 (2018).

[42] R. Agnese et al. (SuperCDMS Collaboration), First Dark Matter Constraints from a SuperCDMS Single-Charge Sensitive Detector, Phys. Rev. Lett. 121, 051301 (2018); Erratum, Phys. Rev. Lett. 122, 069901 (2019).

[43] P. Agnes et al. (DarkSide Collaboration), Constraints on SubGeV Dark-Matter-Electron Scattering from the DarkSide-50 Experiment, Phys. Rev. Lett. 121, 111303 (2018).

[44] M. Settimo (DAMIC Collaboration), The DAMIC experiment at SNOLAB, in 53rd Rencontres de Moriond on Cosmology (ARISF, Paris, 2018), pp. 315-318 [arXiv:1805.10001].

[45] D. Akerib et al. (LUX Collaboration), Results of a Search for Sub-GeV Dark Matter Using 2013 LUX Data, Phys. Rev. Lett. 122, 131301 (2019).

[46] O. Abramoff et al. (SENSEI Collaboration), SENSEI: Direct-Detection Constraints on Sub-GeV Dark Matter from a Shallow Underground Run Using a Prototype SkipperCCD, Phys. Rev. Lett. 122, 161801 (2019).

[47] A. Aguilar-Arevalo et al. (DAMIC Collaboration), Constraints on Light Dark Matter Particles Interacting with Electrons from DAMIC at SNOLAB, Phys. Rev. Lett. 123, 181802 (2019).

[48] E. Aprile et al. (XENON Collaboration), Light Dark Matter Search with Ionization Signals in XENON1T, Phys. Rev. Lett. 123, 251801 (2019).

[49] L. Barak et al. (SENSEI Collaboration), SENSEI: DirectDetection Results on Sub-GeV Dark Matter from a New Skipper-CCD, Phys. Rev. Lett. 125, 171802 (2020).
[50] Q. Arnaud et al. (EDELWEISS Collaboration), First Germanium-Based Constraints on Sub-MeV Dark Matter with the EDELWEISS Experiment, Phys. Rev. Lett. 125, 141301 (2020).

[51] D. Amaral et al. (SuperCDMS Collaboration), Constraints on low-mass, relic dark matter candidates from a surface-operated SuperCDMS single-charge sensitive detector, Phys. Rev. D 102, 091101 (2020).

[52] N. W. Ashcroft and N. D. Mermin, Solid State Physics (Saunders College Publishing, Philadelphia, 1976).

[53] D. Pines, Collective energy losses in solids, Rev. Mod. Phys. 28, 184 (1956).

[54] P. Nozieres and D. Pines, Electron interaction in solids. Characteristic energy loss spectrum, Phys. Rev. 113, 1254 (1959).

[55] H. Raether, Excitation of Plasmons and Interband Transitions by Electrons, Vol. 88 (Springer, New York, 2006).

[56] W. Schülke, Electron Dynamics by Inelastic X-Ray Scattering, Oxford Science Publications (Oxford University Press, New York, 2007).

[57] See Supplemental Material at http://link.aps.org/ supplemental/10.1103/PhysRevLett.127.151802 for additional details and derivations, comparisons of model loss functions to experimental data, and further comparison of our results for superconductors to other results in the literature, which includes Refs. [58-64].

[58] A. Altland and B. D. Simons, Condensed Matter Field Theory (Cambridge University Press, Cambridge, England, 2010).

[59] H. Banks and M. Mccullough, Charting the fifth force landscape, Phys. Rev. D 103, 075018 (2021).

[60] G. D. Mahan, Many-Particle Physics (Springer Science \& Business Media, New York, 2013).

[61] H. Fröhlich, Phenomenological Theory of the Energy Loss of Fast Particles in Solids (VEB Deutscher Verlag der Wissenschaften, Berlin, 1959).

[62] M. E. Peskin and D. V. Schroeder, An Introduction to Quantum Field Theory (Addison-Wesley, Reading, MA, 1995).

[63] J. Mydosh and P. Oppeneer, Hidden order behaviour in $\mathrm{URu}_{2} \mathrm{Si}_{2}$ (a critical review of the status of hidden order in 2014), Philos. Mag. 94, 3642 (2014).

[64] T. T. M. Palstra, A. A. Menovsky, J. van den Berg, A. J. Dirkmaat, P. H. Kes, G. J. Nieuwenhuys, and J. A. Mydosh, Superconducting and Magnetic Transitions in the HeavyFermion System $\mathrm{URu}_{2} \mathrm{Si}_{2}$, Phys. Rev. Lett. 55, 2727 (1985).

[65] Y. Hochberg, T. Lin, and K. M. Zurek, Detecting ultralight bosonic dark matter via absorption in superconductors, Phys. Rev. D 94, 015019 (2016).

[66] Y. Hochberg, T. Lin, and K. M. Zurek, Absorption of light dark matter in semiconductors, Phys. Rev. D 95, 023013 (2017).

[67] I. M. Bloch, R. Essig, K. Tobioka, T. Volansky, and T.-T. Yu, Searching for dark absorption with direct detection experiments, J. High Energy Phys. 06 (2017) 087.

[68] M. Dressel, G. Gruner, and G. Grüner, Electrodynamics of Solids: Optical Properties of Electrons in Matter (Cambridge University Press, Cambridge, England, 2002).

[69] P. Gibbons, S. Schnatterly, J. Ritsko, and J. Fields, Line shape of the plasma resonance in simple metals, Phys. Rev. B 13, 2451 (1976). 
[70] N. Bachar, D. Stricker, S. Muleady, K. Wang, J. A. Mydosh, Y. K. Huang, and D. van der Marel, Detailed optical spectroscopy of hybridization gap and hidden-order transition in high-quality $\mathrm{URu}_{2} \mathrm{Si}_{2}$ single crystals, Phys. Rev. B 94, 235101 (2016).

[71] L. J. Hall, K. Jedamzik, J. March-Russell, and S. M. West, Freeze-in production of FIMP dark matter, J. High Energy Phys. 03 (2010) 080.

[72] X. Chu, T. Hambye, and M. H. Tytgat, The four basic ways of creating dark matter through a portal, J. Cosmol. Astropart. Phys. 05 (2012) 034.

[73] C. Dvorkin, T. Lin, and K. Schutz, Making dark matter out of light: Freeze-in from plasma effects, Phys. Rev. D 99, 115009 (2019).

[74] Y. Sun, H. Xu, B. Da, S.-f. Mao, and Z.-j. Ding, Calculations of energy-loss function for 26 materials, Chin. J. Chem. Phys. 29, 663 (2016).

[75] K. Berggren, Y. Hochberg, Y. Kahn, E. D. Kramer, N. Kurinsky, B. V. Lehmann, and T. C. Yu (to be published).

[76] S. Knapen, J. Kozaczuk, and T. Lin, Migdal Effect in Semiconductors, Phys. Rev. Lett. 127, 081805 (2021).

[77] H.-C. Weissker, J. Serrano, S. Huotari, E. Luppi, M. Cazzaniga, F. Bruneval, F. Sottile, G. Monaco, V. Olevano, and L. Reining, Dynamic structure factor and dielectric function of silicon for finite momentum transfer: Inelastic $\mathrm{x}$-ray scattering experiments and $a b$ initio calculations, Phys. Rev. B 81, 085104 (2010).

[78] J. P. Walter and M. L. Cohen, Frequency-and wave-vectordependent dielectric function for silicon, Phys. Rev. B 5, 3101 (1972).

[79] M. K. Kundmann, Study of semiconductor valence plasmon line shapes via electron energy-loss spectroscopy in the transmission electron microscope, Technical Report, Lawrence Berkeley Laboratory, California, USA, 1988.

[80] T. Wehling, A. Black-Schaffer, and A. Balatsky, Dirac materials, Adv. Phys. 63, 1 (2014).
[81] D. E. Kharzeev, R. D. Pisarski, and H.-U. Yee, Universality of Plasmon Excitations in Dirac Semimetals, Phys. Rev. Lett. 115, 236402 (2015).

[82] J. Hofmann and S. D. Sarma, Plasmon signature in DiracWeyl liquids, Phys. Rev. B 91, 241108(R) (2015).

[83] G. S. Jenkins, C. Lane, B. Barbiellini, A. B. Sushkov, R. L. Carey, F. Liu, J. W. Krizan, S. K. Kushwaha, Q. Gibson, T.-R. Chang et al., Three-dimensional Dirac cone carrier dynamics in $\mathrm{Na}_{3} \mathrm{Bi}$ and $\mathrm{Cd}_{3} \mathrm{As}_{2}$, Phys. Rev. B 94, 085121 (2016).

[84] A. Thakur, R. Sachdeva, and A. Agarwal, Dynamical polarizability, screening and plasmons in one, two and three dimensional massive Dirac systems, J. Phys. Condens. Matter 29, 105701 (2017).

[85] V. Kozii and L. Fu, Thermal plasmon resonantly enhances electron scattering in Dirac/Weyl semimetals, Phys. Rev. B 98, 041109(R) (2018).

[86] G. R. Stewart, Heavy-fermion systems, Rev. Mod. Phys. 56, 755 (1984).

[87] P. S. Riseborough, Heavy fermion semiconductors, Adv. Phys. 49, 257 (2000).

[88] P. Coleman, Heavy fermions and the Kondo lattice: A 21st century perspective, arXiv:1509.05769.

[89] A. J. Millis, M. Lavagna, and P. A. Lee, Plasma oscillations in heavy-fermion materials, Phys. Rev. B 36, 864 (1987).

[90] C. Bareille, F. Boariu, H. Schwab, P. Lejay, F. Reinert, and A. Santander-Syro, Momentum-resolved hidden-order gap reveals symmetry breaking and origin of entropy loss in $\mathrm{URu}_{2} \mathrm{Si}_{2}$, Nat. Commun. 5, 4326 (2014).

[91] O. Krivanek, N. Dellby, J. Hachtel, J.-C. Idrobo, M. Hotz, B. Plotkin-Swing, N. Bacon, A. Bleloch, G. Corbin, M. Hoffman, C. Meyer, and T. Lovejoy, Progress in ultrahigh energy resolution EELS, Ultramicroscopy 203, 60 (2019).

[92] Y. Hochberg, E. D. Kramer, N. Kurinsky, and B. V. Lehmann, Directional detection of light dark matter in superconductors, arXiv:2109.04473.

[93] S. Knapen, J. Kozaczuk, and T. Lin, Dark matter-electron scattering in dielectrics, Phys. Rev. D 104, 015031 (2021). 